\title{
An mHealth intervention to prevent postnatal smoking relapse: The RESPREMO study protocol
}

\author{
Marina Dascal ${ }^{1,2}$, Andreea Rusu ${ }^{1,2}$, Alexandra Onisor ${ }^{1,2}$, Oana Blaga ${ }^{1,2}$, Mckenzie Miller $^{3}$, Cristian Meghea ${ }^{1,2,4}$
}

\begin{abstract}
Up to $70 \%$ of women who quit smoking relapse after birth, usually within 3 months postpartum. The wide adoption of mobile technologies, especially smartphones, in recent years in low- and middle-income countries (LMICs) offers the possibility of low-cost, novel, and innovative mobile phone-based interventions for smoking relapse prevention. This study presents the protocol of the RESPREMO clinical trial for postnatal smoking relapse prevention for enrolled women, who recently gave birth and quit tobacco smoking before or during pregnancy, and their life partners.

This work relies on data collected in two of the largest government-owned obstetrics and gynecology clinics in Cluj-Napoca, Romania. Seventy-five couples were randomized into one of three groups: a) 24 couples were allocated to the first intervention group and asked to install and use the xSmoker app; b) 26 couples were randomized to the second intervention group, who, in addition to the use of the xSmoker app, received text messages with content focused on motivation, problem solving, and dyadic efficacy; and c) 25 couples were randomized into a control group.

Several measures of engagement with the xSmoker app were assessed, including duration of app use, the frequency of utilizing the tool to calculate savings from quitting, number of app-delivered challenges accepted by users, and number of app-delivered cessation and abstinence tips.

If effective, RESPREMO is expected to have a sustainable impact on the prevention of postnatal relapse tobacco smoking with positive effects for both the mother and the newborn. The implications are beyond tobacco control, and relevant to the design and implementation of other mHealth behavioral interventions focused on the pregnancy and reproductive years in general.
\end{abstract}

\section{AFFILIATION}

1 Center for Health Policy and Public Health, College of Political, Administrative and Communication Sciences, Babes-Bolyai University, Cluj-Napoca, Romania

2 Department of Public Health, College of Political, Administrative and Communication Sciences, Babes-Bolyai University, ClujNapoca, Romania

3 College of Human Medicine, Michigan State University, East Lansing, United States

4 Department of Obstetrics, Gynecology and Reproductive Biology, College of Human Medicine, Michigan State University, East Lansing, United States

\section{CORRESPONDENCE TO}

Andreea Rusu. Center for Health Policy and Public Health, College of Political, Administrative and Communication Sciences, BabesBolyai University, 7 Pandurilor Street, Cluj-Napoca, 400376, Romania. E-mail:

andreea.hostina@publichealth.ro

\section{KEYWORDS}

RCT, mHealth, couples intervention, app, smoking relapse prevention

Received: 6 January 2020

Revised: 28 February 2020

Accepted: 3 March 2020

\section{INTRODUCTION}

Tobacco cigarette smoking remains the leading global cause of preventable disease and the death of nearly 8 million people annually, with most of these deaths occurring in low- and middle-income countries (LMICs) such as Romania ${ }^{1}$. Maternal smoking is one of the most modifiable risk factors clearly linked to adverse effects for the fetus and baby ${ }^{2}$. Preconception and pregnancy smoking are high in Romania and there is a need for local adaptations of tobacco interventions ${ }^{3-5}$. Prior research estimates a $30 \%$ smoking prevalence rate among Romanian women during preconception ${ }^{6}$ with approximately half quitting early in the gestational period. A significant 
problem, and the focus of this project, is that up to $70 \%$ of women who quit smoking relapse after birth, usually within 3 months postpartum ${ }^{7}$.

One difficulty in preventing smoking relapse is that motivation to stay smoke-free after quitting fluctuates over time. In addition, one of the factors that influences smoking cessation during pregnancy and smoking relapse after birth is living with a smoking partner ${ }^{8}$. In Romania, up to $80 \%$ of pregnant smokers have a partner who is also smoking ${ }^{9}$. Preliminary work suggests that couple-oriented interventions, with particular focus on the dyadic efficacy for smoking cessation ${ }^{10}$, may be successful in preventing smoking relapse.

The wide adoption of mobile technologies, especially smartphones ${ }^{11}$, in recent years in LMICs offers the possibility of low-cost, novel, and innovative mobile phone-based interventions for smoking relapse prevention ${ }^{12,13}$. Mobile phones offer a virtually unused opportunity to deliver tobacco cessation interventions, particularly relevant in LMICs characterized by underfunded and understaffed health systems. Mobile health (mHealth) intervention benefits include scalability to large populations, the ability to tailor content to key user characteristics (such as motivation, selfefficacy, demographics), the ability to send timesensitive messages, and the delivery of content that can distract the user from cravings ${ }^{14}$.

Some successful pre- and postnatal smoking prevention interventions have been implemented ${ }^{15-17}$ and nearly all in high-income countries, but most did not include partner support and the few that did were generally not successful in reducing pregnancy smoking and relapse ${ }^{18,19}$. The theoretical relapse prevention model suggests that effective programs for preventing postpartum smoking relapse need to combine enhancing the motivation to maintain abstinence with developing the self-efficacy to do so $^{16}$. An intervention combining these two components, the Motivation and Problem Solving (MAPS) approach, showed promise in preventing postnatal smoking relapse among low-income US women who spontaneously quit during pregnancy ${ }^{16}$ and is currently being tested for pregnancy smoking cessation efficacy in Romania.

Most of the tested mHealth smoking cessation applications do not follow clinical guidelines ${ }^{20,21}$, do not draw on behavioral change theories, and very few have been implemented in LMICs such as Romania ${ }^{14}$. Few smoking cessation mHealth interventions used apps, and there is only one pregnancyspecific app that has been tested in a large-scale randomized controlled trial (RCT) ${ }^{22}$. This study presents the protocol of the RESPREMO three-arm clinical trial for postnatal tobacco smoking relapse prevention. The specific objectives of the RCT are: 1) to develop an adapted and enhanced mHealth couples intervention to prevent postpartum smoking relapse based on the xSmoker mobile application enhanced with SMS-delivered MAPS-based content informed by our work with the target population in Romania; 2) to conduct a pilot-test-scale randomized controlled trial (RCT) of the intervention; and 3) to examine in the pilot test the implementation feasibility and initial efficacy of the intervention in reducing maternal smoking relapse after birth. The study was approved by the Babes-Bolyai University Ethics Committee.

\section{METHODS}

\section{Setting}

The setting for the data collection included the two largest government-owned obstetrics and gynecology clinics located in Cluj-Napoca, Romania: Clinica Obstetrica-Ginecologie I and Clinica ObstetricaGinecologie II. After Bucharest, Cluj-Napoca is considered the second largest metropolitan area, with over 420000 residents, and an important university center of Romania.

\section{Participants}

Eligible participants in the RCT included mothers who: 1 ) were $\geq 18$ years (age of legal tobacco consumption in Romania); 2) quit smoking in the 6 months before pregnancy or during pregnancy and remained quit, biochemically verified at the time of their enrollment in the RCT through exhaled CO $(<4$ ppm); 3) were married or having a stable partner; 4) owned a mobile phone capable to install apps; 5) were willing to offer the contact details of their life partner for the project team to contact them and invite them to participate in the study; and 6) were willing to sign the RCTs informed consent. A total of 75 women were included in the study irrespective of the smoking status of the partner/husband or his decision 
Figure 1. RESPREMO clinical trial CONSORT flow diagram

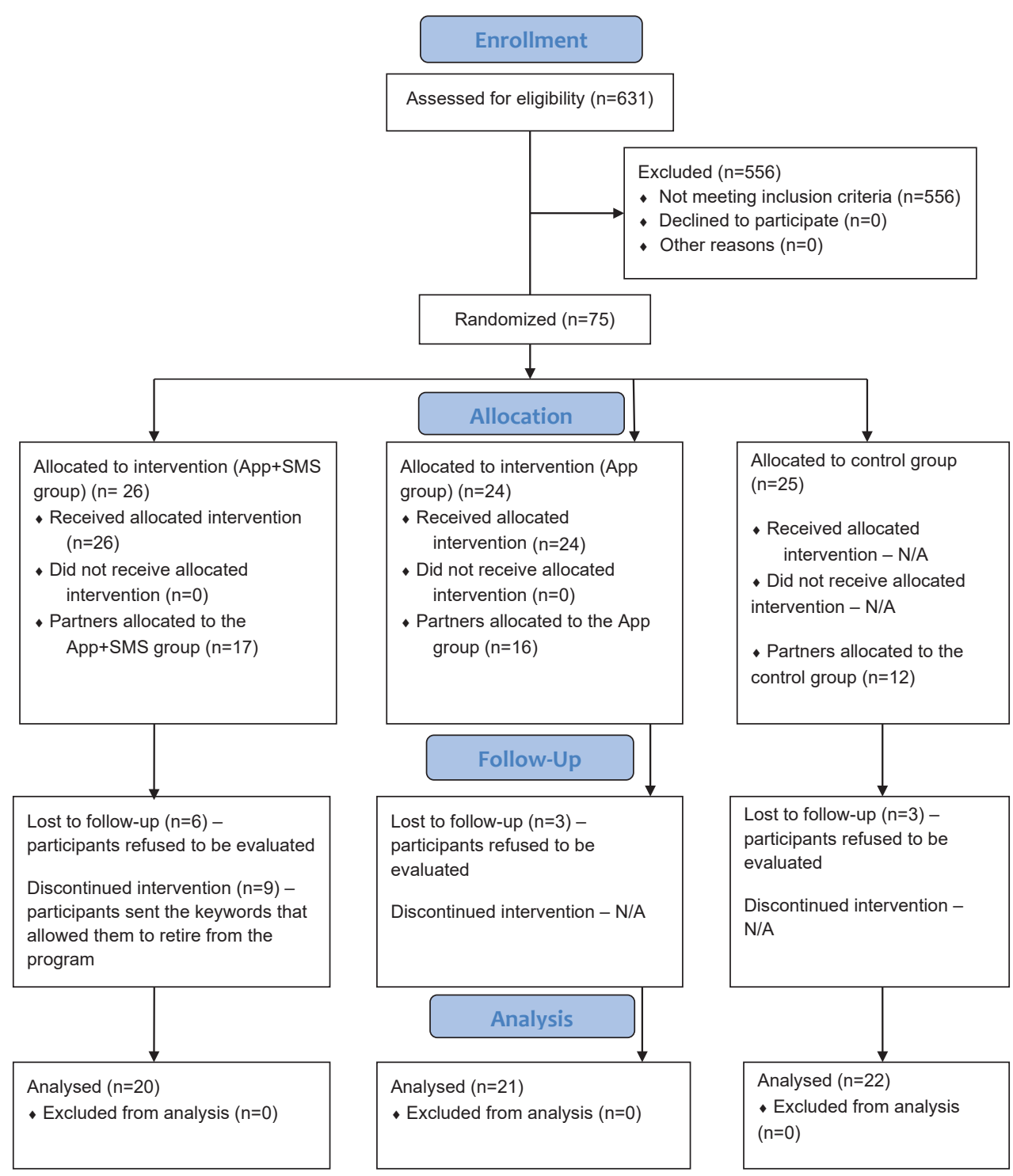

to participate in the RCT. Partners were contacted and invited to take part in the RCT in the first week after the woman's enrollment. If the partners were willing to participate in the RCT, a verbal consent was obtained over the phone by a data collector trained in the study's standard operating procedures. A total of 43 partners agreed to participate in the RCT.

\section{Recruitment of the participants}

The recruitment of the participants in the RCT took place between June and November 2018. The new mothers were approached by a data collector in the maternity wards of the two obstetrics and gynecology clinics in the first days after they gave birth. The data collector briefly described the research study, allowed women to ask questions about the study, and assessed the women's eligibility before asking them to sign two copies of an informed consent. Next, the data collector asked for their contact details (names, phone numbers, and e-mail addresses) and of their partner. Women were invited to fill in an electronic version of the RCT's baseline questionnaire by using a tablet with internet connection. Women who did not feel comfortable filling in the survey by themselves were assisted by the data collector. The baseline questionnaire for women consisted of five sections.

\section{Sociodemographic information}

Ten general questions regarding age, level of education, ethnicity, residence, monthly net income and others. 


\section{Medical and reproductive history}

Nine questions related to the number of pregnancies so far, and previous pregnancy complications.

\section{Information on smoking and alcohol use}

Eighteen questions related to smoking behavior, exposure to passive smoking, previous smoking cessation initiatives, and alcohol or drug use.

Information on the relationship with the spouse/partner Questions regarding the relationship with the partner, as well as his smoking status.

\section{Emotional health}

Two questions that relate to recent emotional experiences.

After completing the questionnaire, the data collector offered all women who were enrolled an informative leaflet on the benefits of remaining smoke-free after birth, for both the mother and the baby, the identification and prevention of risky situations that might lead to smoking relapse, examples of coping strategies that might help them overcome these situations, as well as information on how their life partner can support them throughout the process of remaining smoke-free. As a token of appreciation for their interest and participation in the research study, each participant received a baby nasal aspirator worth $\$ 5$.

\section{Randomization}

Assignment in the RCT arm was done by the data collector in the clinic immediately after each woman's enrollment in the RCT, based on a pre-randomized list of unique participant identification codes. The codes have been pre-randomized into two intervention arms and one control arm by using a blocked randomization procedure with randomly selected block sizes, to avoid selection bias and achieve sample size balance across RCT arms $^{23}$. A total of 75 women were randomized into one of the three groups, with subsequent attempts to enroll their life partners as well: 24 were allocated to the first intervention group and asked to install and use the xSmoker app; 26 were randomized to the second intervention group who in addition to the use of the xSmoker app also received SMS text messages with content based on the MAPS framework and dyadic efficacy and informed by our prior work ${ }^{24,25}$ for 6 weeks; and 25 were assigned to the usual care group. The life partners were allocated in the same groups as the enrolled women.

\section{Control arm}

The participants in the control arm ('usual care') did not receive any intervention apart from the leaflet outlining the benefits of and advice on tobacco abstinence that every participant in the RCT received at recruitment.

\section{Intervention arms}

The development of the RESPREMO smoking relapse prevention intervention was guided by MAPS principles and dyadic efficacy and was informed by our previous work ${ }^{24,25}$. The dyadic efficacy for smoking cessation is a new concept defined as 'an individual's perceptions of confidence about his/her shared abilities to work together with a partner to quit smoking and manage the emotional and practical challenges associated with quitting ${ }^{10}$. The delivery of the mHealth intervention began immediately after birth and continued 6 weeks postpartum, for both women and their partners, according to the study group to which the couple was randomly assigned: a) xSmoker - mHealth intervention using the app $\mathrm{xSmoker}$; and b) xSmoker+ - intervention using the xSmoker app and phone text messages with content based on the Motivation and Problem Solving approach informed by our work ${ }^{24,25}$. The participants (women and life partners) randomized to the intervention arms were either assisted by the data collector in the clinic to install the xSmoker app on their smartphone or they had the chance to install it themselves.

The xSmoker app was developed with European Commission funding. The app xSmoker is a digital health coaching mobile app that helps individuals stop smoking and remain smoke-free. The initial version of the $x$ Smoker application was received from the developers and translated into Romanian by the research team. The content has been divided into three main sections.

\section{Daily tips}

These were approximately 630 items that included information on the beneficial effects of quitting smoking, e.g. 'You have probably already felt very many benefits since you stopped smoking. Your health 
has improved every day. Your home is cleaner, you have better blood circulation and you have a lower likelihood of serious heart diseases. You also have a better self-image, more self-confidence and a better feeling of control over your life! So, do you still have a reason to start smoking again?'.

\section{Panic tips}

These were approximately 100 items that could be accessed at times when the risk of smoking relapse is high, e.g. 'Having a difficult time? Go for a walk, drink a glass of water or play a game on your phone! Seeking a distraction sounds simple, but it works!.

\section{Library}

These were about 120 items that contained detailed information on the topics included in the first two sections, e.g. 'Smokers who prepare to stop smoking have more chance of succeeding than smokers who stop impulsively and without thinking. One way to prepare well is to gradually reduce the smoking habit until the smoking threshold is very low.'.

At the beginning of the intervention, participants that were enrolled in one of the intervention groups were encouraged to use all the features of the app, besides the above mentioned main categories, that also included a calculator of the money saved since they stopped smoking and different games and challenges that aim to keep the motivation to remain smoke-free at the highest level. The app also had a so-called 'panic button', which the participants were encouraged to push if they smoked or if they felt the urge to smoke. If this happened, the app immediately prompted a message from the Panic tips that would help the participant get over that moment. Both women and their life partners were instructed to install and use the app, which was directly relevant to women (all of whom quit smoking prior to or during pregnancy) and to their life partners who smoked or recently quit smoking.

The app was enhanced with SMS-delivered content addressing the dyadic efficacy and using the MAPS (Motivation and Problem Solving) framework for smoking cessation aiming to improve both partners skills to work as a team to support the prevention of the woman's postpartum smoking relapse. The content of the messages was based on the 6-year relapse smoking prevention experience of the research team ${ }^{24,25}$ and on the MAPS framework ${ }^{16}$, and was enhanced with content supporting dyadic efficacy. MAPS is a relatively new approach to substance use treatment combining motivational enhancement and social cognitive methods based on Motivational Interviewing (MI), the Treating Tobacco Use and Dependence Clinical Practice Guideline ${ }^{21}$ and social cognitive/relapse prevention theory ${ }^{26}$. MAPS addresses a weakness in social cognitive/relapse prevention treatments for tobacco smoking cessation, specifically the lack of focus on the fluctuating nature of motivation, by embedding practical problem-solving strategies within a motivational framework, dynamically switching between skills training and motivational enhancement based on motivation to attempt, achieve, and maintain abstinence ${ }^{16}$.

The development of the SMS text messages was led by AO, the co-author with MI expertise culturally adapted to Romania, currently a member of the Motivational Interviewing Network of Trainers (MINT). Six categories of messages were developed and sent to participants: 1) Importance and trust, 2) Fear of relapse, 3 ) Partner support, 4) Breastfeeding, 5) The need to smoke, and 6) Relapse. Four major objectives were established based on the content of the SMS text messages, with the help of the literature: 1) Supporting motivation, e.g. 'Staying smoke-free after birth helps you have an increased level of energy and cope with this busy period. What benefits do you feel?'; 2) Supporting self-efficacy, e.g. 'Good morning! There has been a lot of changes in your life lately. How do you face them? How could you relax for a few minutes daily?'; 3) Supporting dyadic efficacy, e.g. 'Good morning! The baby is very important for you but so is the relationship with your partner. What would help you so that you could also spend time with your partner?'; and 4) Developing problem-solving skills, e.g. 'When you are in a place where people smoke, you can talk to your partner about something else, to draw your attention from them. How does this solution sound?'.

The content of the messages was developed using MI techniques, including the importance/confidence ruler, the decisional balance, looking forward, and goals and values. Various themes were addressed, including the benefits of tobacco abstinence for mother and baby, passive smoking, staying healthy, 
and having healthy children. Four categories of questions answered by the participants tailored the content of the received text messages: the importance of staying abstinent, the confidence of staying abstinent, coping with relapse situations, and whether the participants lapsed or relapsed smoking.

The SMSs sent to the women's life partners focused on increasing the woman's motivation to stay smokefree (e.g. 'It is important to you to have a healthy child. Helping the mother to stay smoke-free protects the child from asthma, respiratory infections, and pulmonary disease.'), increasing his confidence in helping the ex-smoker mother stay smoke-free (e.g. 'What could you do when you see that your partner is tempted to light up a cigarette? How do you feel about talking to her about strategies to avoid smoking that you could develop together? You would be more prepared to resist the temptations.'), and improving her coping with situations at risk for relapse.

The messages were delivered using Textit, a platform for visually building interactive SMS applications (htpps://textit.in). All the messages were uploaded in the platform and different flows and subflows were created for every day of the intervention to automate the process of SMS delivery. A combination of trigger words and skip patterns was used in order to tailor the messages, e.g. the participants could send the word 'STOP' at any point if they wanted to stop receiving messages. The two-way interaction offered the participants the opportunity to reply to the received SMSs with numbers or words, depending on the stage of the intervention they were in. Each participant received between two and six messages daily, for the six weeks duration of the intervention. The SMSs content was tailored separately for women and their partners and the messages were sent at different times throughout the day.

\section{Primary outcomes}

Feasibility measures included uptake rates, engagement with the app and SMS texts, and followup rates in the interventions arm, separately among women and their partners. Seven-day point prevalence abstinence (PPA) and prolonged abstinence (PA) among women will be the primary outcomes assessed at 3 months post-birth. Regarding PPA, women who reported no smoking, not even a puff in the previous
7 days, were mailed a saliva cotinine test and asked to send at least one photo of the completed test. Those with a salivary cotinine $<10 \mathrm{ng} / \mathrm{mL}$ were considered confirmed non-smokers. PA was defined based on the question 'Have you smoked tobacco cigarettes over the past 6 months', with PA failure determined by the answers: 'Yes, I smoked, but never a whole cigarette'; 'Yes, I smoked cigarettes, but only occasionally'; or 'Yes, I smoked cigarettes daily for a period (or periods) of time'.

\section{Intervention engagement}

Several measures of app engagement with xSmoker were assessed including: duration of app use, defined as the number of days between first and last use; the frequency of utilizing the tool to calculate savings from quitting; number of app-delivered challenges accepted by users; and number of app-delivered cessation and abstinence tips. Measures of receipt and engagement with the SMS text messages included the number of weeks receiving messages, number of messages received, number of replies from users, and whether users opted out from receiving messages.

\section{Statistical analysis}

To assess feasibility, we will analyze group differences in mean satisfaction, duration, mean and frequencies of the xSmoker app use, as well as follow-up rates, along with mixed adjusted multivariate models addressing randomization failures by including the unbalanced baseline variables as covariates. Regarding the efficacy of xSmoker and xSmoker+, we will employ intentto-treat techniques for all intervention analyses with those lost to follow-up considered relapsed smokers. Distinct analyses will be performed for women and life partners. To test our primary treatment effect hypotheses, we will employ multivariate analyses including random effects regression models (subjectspecific effects) and the method of generalized estimating equations (population-averaged effects, fewer assumptions) modeled including the effect of time within participant. All efficacy analyses will include models that will account for the engagement with the app and the receipt of SMS text messages.

\section{DISCUSSION}

RESPREMO has the potential to identify effective strategies to prevent postnatal tobacco smoking relapse 
among women who spontaneously quit smoking before or during pregnancy and are smoke-free at the time they are giving birth. This contribution will be significant because understanding the effectiveness of such an intervention will help health systems design and adopt successful smoking prevention campaigns targeted to couples throughout their reproductive life. The work proposed here sets the foundation for a program of research on dyadic efficacy and smoking cessation, potentially moving forward the field of relapse prevention research. This research is expected to have a significant impact on the methodology and design of clinical and public health interventions during the reproductive years.

Our results have immediate relevance to the Romanian health system, and potentially other health systems. Applications of our research may include mechanisms to integrate and sustain the postpartum smoking relapse intervention in the Romanian health system. The proposed intervention adaptation and mHealth delivery will represent groundwork for building a comprehensive implementation support system for the future scaling up of this pilot intervention. If effective, our intervention could have a major public health impact because it has a large potential for dissemination and adoption into the Romanian national 'STOP SMOKING' program that includes a quitline. We anticipate strong potential for the future proactive implementation of the proposed mHealth intervention for couples referred by prenatal health providers, as an extension of the existing quitline in the Romanian universal public health system. Interventions that minimize clinic-based face-to-face contact, such as mHealth approaches, may be useful in increasing the dosage of treatment and improving adherence ${ }^{16}$. Proactive quitlines are recommended based on strong evidence of effectiveness in increasing tobacco cessation ${ }^{10}$. While the focus is using pregnancy and the reproductive years as a window of opportunity for couples to remain smoke-free, the approach is relevant for delivering other interventions during the reproductive years.

The RCTs evaluation, currently under way, will report the effects of the RESPREMO intervention on: rates of prolonged and point prevalence maternal smoking abstinence biochemically verified via salivary cotinine, maternal motivation, self-efficacy, dyadic efficacy for smoking cessation, spousal smoking abstinence, and cigarettes per day among those who smoke.

\section{CONCLUSIONS}

If effective, RESPREMO is expected to have a sustainable impact on the prevention of postnatal relapse in tobacco smoking with positive effects for both the mother and the newborn. The implications are beyond tobacco control, and relevant to the design and implementation of other mHealth behavioral interventions focused on the pregnancy and reproductive years in general.

\section{REFERENCES}

1. World Health Organization. WHO report on the global tobacco epidemic 2019. https://www.who.int/tobacco/ global_report/en/. Accessed January 6, 2020.

2. Keegan J, Parva M, Finnegan M, Gerson A, Belden M. Addiction in Pregnancy. J Addict Dis. 2010;29(2):175191. doi:10.1080/10550881003684723

3. Meghea CI, Rus D, Rus IA, Summers Holtrop J, Roman L. Smoking during pregnancy and associated risk factors in a sample of Romanian women. Eur J Public Health. 2012;22(2):229-233. doi:10.1093/eurpub/ckq189

4. Al-Sahab B, Saqib M, Hauser G, Tamim H. Prevalence of smoking during pregnancy and associated risk factors among Canadian women: a national survey. BMC Pregnancy Childbirth. 2010;10(1):24. doi:10.1186/1471-2393-10-24

5. World Health Organization. Global adult tobacco survey: Romania 2018. Geneva, Switzerland: World Health Organization; 2018.

6. Blaga OM, Brînzaniuc A, Rus IA, Cherecheș RM, Wallis AB. Smoking and Smoking Cessation During Pregnancy. An Analysis of a Hospital Based Cohort of Women in Romania. J Community Health. 2017;42(2):333-343. doi:10.1007/s10900-016-0259-6

7. Winickoff JP, Healey EA, Regan S, et al. Using the postpartum hospital stay to address mothers and fathers smoking: the NEWS study. Pediatrics. 2010;125(3):518525. doi:10.1542/peds.2009-0356

8. Schneider S, Huy C, Schuetz J. Smoking cessation during pregnancy: a systematic literature review. Drug alcohol. 2010;29(1):81-90. doi:10.1111/j.1465-3362.2009.00098.x

9. Meghea CI, Rus IA, Rus D. Risk factors associated with nicotine dependence in a sample of Romanian pregnant smokers. Eur J Obstet Gynecol Reprod Biol. 2012;163(1):22-26. doi:10.1016/j.ejogrb.2012.03.033

10. Sterba KR, Rabius V, Carpenter MJ, Villars P, Wiatrek D, McAlister A. Dyadic Efficacy for Smoking Cessation: Preliminary Assessment of a New Instrument. Nicotine Tob Res. 2011;13(3):194-201. doi:10.1093/ntr/ntq236 
11. GSM Association. Ericsson Mobility Report: On the pulse of the Networked Society. https://www.gsma. com/latinamerica/ericsson-mobility-report-networkedsociety/. Published August 30, 2013. Accesses January 6, 2020.

12. Lopéz DM, Blobel B. MHealth in low- and middleincome countries: Status, requirements and strategies. Stud Health Technol Inform. 2015;211:79-87. PMID:25980851.

13. Kaplan WA. Can the ubiquitous power of mobile phones be used to improve health outcomes in developing countries? Global Health. 2006;2(1):9. doi:10.1186/1744-8603-2-9

14. Marcolino MS, Oliveira JAQ, DAgostino M, Ribeiro AL, Alkmim MBM, Novillo-Ortiz D. The Impact of mHealth Interventions: Systematic Review of Systematic Reviews. JMIR mHealth uHealth. 2018;6(1):e23. doi:10.2196/mhealth.8873

15. Hajek P, Stead LF, West R, Jarvis M, Hartmann-Boyce J, Lancaster T. Relapse prevention interventions for smoking cessation. Cochrane database Syst Rev. 2013;8:CD003999. doi:10.1002/14651858.CD003999.pub4

16. Reitzel LR, Vidrine JI, Businelle MS, et al. Preventing postpartum smoking relapse among diverse low-income women: a randomized clinical trial. Nicotine Tob Res. 2010;12(4):326-335. doi:10.1093/ntr/ntq001

17. Lumley J, Chamberlain C, Dowswell T. Interventions for promoting smoking cessation during pregnancy. Database Syst Rev. 2009;(3):CD001055. doi:10.1002/14651858.CD001055.pub3

18. Hemsing N, Greaves L, OLeary R. Partner support for smoking cessation during pregnancy: a systematic review. Nicotine Tob. 2011;14(7):767-776. doi:10.1093/ntr/ntr278

19. McBride CM, Baucom DH, Peterson BL, et al. Prenatal and postpartum smoking abstinence a partner-assisted approach. Am J Prev Med. 2004;27(3):232-238. doi:10.1016/j.amepre.2004.06.005

20. Abroms LC, Lee Westmaas J, Bontemps-Jones J, Ramani R, Mellerson J. A content analysis of popular smartphone apps for smoking cessation. Am J Prev Med. 2013;45(6):732-736. doi:10.1016/j.amepre.2013.07.008

21. Clinical Practice Guideline Treating Tobacco Use and Dependence 2008 Update Panel, Liaisons, and Staff. A clinical practice guideline for treating tobacco use and dependence: 2008 update. A U.S. Public Health Service report. Am J Prev Med. 2008;35(2):158-176. doi:10.1016/j.amepre.2008.04.009

22. Tombor I, Beard E, Brown J, Shahab L, Michie S, West R. Randomized factorial experiment of components of the SmokeFree Baby smartphone application to aid smoking cessation in pregnancy. Transl Behav Med. 2018;9(4):583-593. doi:10.1093/tbm/iby073

23. Efird J. Blocked randomization with randomly selected block sizes. Int J Environ Res Public Health. 2011;8(1):15-20. doi:10.3390/ijerph8010015
24. Brinzaniuc A, Strilciuc A, Blaga OM, Chereches RM, Meghea CI. Smoking and quitting smoking during pregnancy: A qualitative exploration of the socio-cultural context for the development of a couple-based smoking cessation intervention in Romania. Tob Prev Cessation. 2018;4(March). doi:10.18332/tpc/86161

25. Meghea C, Brinzaniuc A, Sidor A, Chereches R, Mihu D, Iuhas C, et al. A couples-focused intervention for smoking cessation during pregnancy: The study protocol of the Quit Together pilot randomized controlled trial. Tob Prev Cessation. 2018;4(May). doi:10.18332/tpc/89926

26. Witkiewitz K, Marlatt GA. Relapse prevention for alcohol and drug problems: that was Zen, this is Tao. Am Psychol. 59(4):224-235. doi:10.1037/0003-066X.59.4.224

\section{CONFLICTS OF INTEREST}

The authors have completed and submitted the ICMJE Form for Disclosure of Potential Conflicts of Interest and none was reported.

\section{FUNDING}

This work was supported by a grant of the Ministry of Research and Innovation, CNCS-UEFISCDI, project number PN-III-P4-ID-PCE-2016-0632, within PNCDI III.

\section{PROVENANCE AND PEER REVIEW}

Not commissioned; externally peer reviewed. 